\title{
A study on maternal near miss cases in Government Medical College Shivpuri, India
}

\author{
Uma Jain* \\ Department of Obstetrics and Gynecology, Government Medical College Shivpuri, Madhya Pradesh, India
}

Received: 30 June 2019

Revised: 03 July 2019

Accepted: 08 July 2019

\section{*Correspondence:}

Dr. Uma Jain,

E-mail: Ujain880@gmail.com

Copyright: ( $\odot$ the author(s), publisher and licensee Medip Academy. This is an open-access article distributed under the terms of the Creative Commons Attribution Non-Commercial License, which permits unrestricted non-commercial use, distribution, and reproduction in any medium, provided the original work is properly cited.

\begin{abstract}
Background: Obstetrics near miss is an important indicator that reflects the quality of obstetrics care in a health facility. It assesses and monitors the activities aimed for prevention of maternal mortality. The aim and objective of this study was to find out the incidence, the prevalence and causes of maternal near miss due to severe obstetric complications and to identify the gapes and contextualize corrective measures to be taken in our facility.

Methods: This is a retrospective study done in department of Obstetrics and Gynecology in GMC associated with DHS Shivpuri MP. The study was done during a period from 1st January 2018 to 30 April 2019.

Results: In this study the hospital maternal near miss incidence ratio was $14.34 \%$. In our study we found the most common morbidity was (30.18\%) hypertensive disorder of pregnancy. These 159 near miss diagnoses were comprised of $(30.18 \%)$ cases of Hypertensive disorder of pregnancy, (27.67\%) cases of major obstetric hemorrhage, (6.91) Severe systemic infection or sepsis, $(4.40 \%)$ Labour related disorders. In Medical disorders very Severe Anemia, (1.88\%) was most common cause of near miss. The most common cause of death was post-partum hemorrhage $37.5 \%$ and most of the patients referred from periphery in very critical condition. The median time taken to get clinical intervention among cases was 20-40 minutes after admission.

Conclusions: Hemorrhage and hypertension disorders are the leading causes of MNM. Prompt diagnosis and adequate management of near miss cases can reduce mortality rates.
\end{abstract}

Keywords: Hypertensive disorders, Maternal mortality, Obstetric hemorrhage, Severe acute maternal morbidity

\section{INTRODUCTION}

Maternal mortality is a critical indicator to assess the quality of services provided by a health care system. Women who experienced and survived a severe health condition during pregnancy, childbirth or postpartum are considered as near miss or severe acute maternal morbidity cases. ${ }^{1}$

The major reasons and causes are the same for both MNM and MDR, so review of MNM cases is likely to yield valuable information regarding severe morbidity which could lead to death of mother, if not intervened properly and in time. According to WHO "a woman who survives life threatening conditions during pregnancy, abortion and childbirth or within 42 days of pregnancy termination, irrespective of receiving emergency medical/surgical interventions, is called maternal near miss". 2

By reviewing near miss cases, we can learn about the processes and their deficiencies that are in place for the 
care of pregnant women. This would result in identifying the pattern of severe maternal morbidity and mortality, strengths and weakness in the referral system and the clinical interventions available and the ways in which improvements can be made. ${ }^{3}$

In order to improve obstetric care and reduce delay, critical incident audit and feedback have been recommended by the world health organization (WHO) and other institutions.

When MNMR is used in conjunction with MDR- (i) Aids in recognizing patterns and trends of maternal morbidity and mortality (ii) Helps in identifying contributory factors of maternal deaths so that actions can be taken at various levels. (iii) Assists in evaluation of quality of health care at facility and to monitor it.(iv) Facilitates detection of lacunae in existing system.(v) Helps in setting up a database to capture all locations and facility details to identify where an MNM case comes from; this assists in focusing interventions in a particular location. (vi)

Beneficial in assessing and analyzing requirement of health care facility in terms of interventional facilities, in terms of infrastructure, human resource and interventional facilities, besides comparing the existing health care and optimal health care of a facility. (vii) Identification of delays at various levels can be done. Which lead to maternal morbidity and mortality. (viii) Identify modifiable socio-demographic factors responsible for maternal morbidity and mortality. (xi) It assists in international comparisons in imparting optimal health care. ${ }^{4}$

The objective of this study to find out the socio demographic factors associated with maternal near miss cases, to determine the prevalence of maternal near miss due to severe obstetric complications and to identify the gapes and contextualize corrective measures to be taken in this facility.

\section{METHODS}

This is a retrospective study done in department of Obstetrics and Gynecology in GMC associated with DHS Shivpuri MP. The study was done during a period from 1st January 2018 to 30 April 2019. GMC associated with
DHS Shivpuri is a 350 bedded hospital. This is a referral hospital. It provides 24 Hour emergency obstetric service for both low and high risk pregnant women. Data were collected from the medical records and Data of computer operator of Maternity wing.

For each case Booking, literacy, socio- economic status, gestational age, parity, referred or self-referred, mode of delivery, disease responsible for critical illness, nature of obstetric complications, presence of organ and/or system dysfunction, duration of hospital stays, and source of referral, requirement of Blood, surgical intervention to save the life of mother and other relevant information were collected from the medical records and Data of computer operator of Maternity wing.

Different causes of maternal near miss were indentified according to maternal near miss review operational guideline - India Dec 2014. (Table 1). All the data was analyzed using IBM SPSS ver 20.

Software Frequency distribution and cross tabulation was used to prepare tables, data is expressed as percentage. According to our infrastructure and setting I choose some disease specific criteria and included pregnant and recently delivered women up to 6 weeks postpartum with one or more of the following entities:

- $\quad$ Severe hypertension and hypertensive emergency are identified according to maternal near miss review operation guideline Dec 2014.

- Hemorrhage leading to shock; emergency hysterectomy; coagulation defects and / or blood transfusion of $\geq 2$ liters in our setting.

- Uterine rupture, defined as the occurrence of clinical symptoms pain, fetal distress, acute loss of contractions, hemorrhage or intrauterine fetal death, that led to laparotomy, at which the diagnosis was confirmed, or laparotomy for uterine rupture after vaginal birth.

- Severe sepsis according to maternal near miss review operation guideline Dec 2014.

- Very severe anemia- I take very severe anemia cases of $\mathrm{Hb}<6 \mathrm{gm} / \mathrm{dl}$ in my study who require $\geq 3$ units of blood or PRBCs and multiple dose of injectable iron.

Table 1: Criteria for Identification of MNM Cases (Maternal near miss review operational guidelineIndia Dec 2014). 5

For diagnosis of near Miss, the patient should meet minimum 3 criteria: one each from 1) clinical findings (either symptoms or signs, 2) investigations and 3) interventions done or any single criteria which signifies cardiorespiratory collapse

\begin{tabular}{|llll|}
\hline Haemorrhage & & & \\
\hline $\begin{array}{l}\text { Abortion, } \\
\text { Ruptured ectopic, }\end{array}$ & $\begin{array}{l}\text { Any bleeding from } \\
\text { or into the genital }\end{array}$ & $\begin{array}{l}\text { Altered conscious state } \\
\text { Tachycardia }>120 / \mathrm{min}\end{array}$ & $\begin{array}{l}\text { Acute fall } \mathrm{Hb}<5 \text { gm } \% \text { or } 30 \% \text { fall in } \\
\text { haematocrit }\end{array}$ \\
\hline
\end{tabular}




\begin{tabular}{|c|c|c|c|}
\hline \multicolumn{4}{|c|}{$\begin{array}{l}\text { For diagnosis of near Miss, the patient should meet minimum } 3 \text { criteria: one each from 1) clinical findings } \\
\text { (either symptoms or signs, 2) investigations and } 3 \text { ) interventions done or any single criteria which signifies } \\
\text { cardiorespiratory collapse }\end{array}$} \\
\hline $\begin{array}{l}\text { APH } \\
\text { Placent previa } \\
\text { Placent abruption } \\
\text { Rupture uterus } \\
\text { Surgical injury, } \\
\text { III stage compli., } \\
\text { inversion of } \\
\text { uterus, retained } \\
\text { placent, cervical } \\
\text { tear, } \\
\text { PPH }\end{array}$ & $\begin{array}{l}\text { tract leading to } \\
\text {-Air hunger } \\
\text {-Sycopal attacks }\end{array}$ & $\begin{array}{l}\text { Low volume pulse } \\
\text { Bradycardia }<40 / \mathrm{min} \\
\text { Tachypnea }>40 / \mathrm{min} \\
\text { Bradypnea }<6 / \mathrm{min} \\
\text { Blood pressure, } \\
\text { Systolic }<90 \mathrm{mmHg}, \\
\text { Diastolic }<60 \mathrm{mmHg} \\
\text { Absent peripheral relexes } \\
\text { Oliguria with output } \\
\text {,30ml/ hour }\end{array}$ & $\begin{array}{l}\text { (fall in hemoglobin so as to affect oxygen } \\
\text { saturation), Fall in oxygen saturation below } \\
90 \% \\
\mathrm{PaO} 2 \text { : } \mathrm{FiO} 2<200, \mathrm{PaCO} 2>50 \mathrm{mmHg} \\
\text { Platelet }<20000 \text { (Acute Decline in platelet } \\
\text { count more significant } \\
\text { Clot observation time }>7 \text { min or any other } \\
\text { test done which proves deranged } \\
\text { coagulation profile } \\
\text { Serum cratinine }>3.5 \mathrm{mg} / \mathrm{dl} \\
\text { ECG -Ischemic changes, ST inversion } \\
\text { elevation }\end{array}$ \\
\hline \multicolumn{4}{|l|}{ Hypertension } \\
\hline $\begin{array}{l}\text { SEVERE (PIH) } \\
\text { Preeclampsia, } \\
\text { Eclampsia, } \\
\text { HELLP Syndrome }\end{array}$ & $\begin{array}{l}\text { Convulsions, } \\
\text { diminution/ } \\
\text { Blurring of viosion, } \\
\text { severe epigastric } \\
\text { pain, severe } \\
\text { headache non } \\
\text { responsive to pain } \\
\text { killers, difficulty in } \\
\text { breathing, } \\
\text { palpitations }\end{array}$ & $\begin{array}{l}\text { Altered conscious state, } \\
\text { BP } \geq 160 / 110 \mathrm{mmHg}, \\
\text { Deep Jaundice, } \\
\text { Oliguria/ anuria/ } \\
\text { haematuria, Coma, } \\
\text { Coagulation failure, } \\
\text { Pulmonary edema, } \\
\text { Evidence of circulatory } \\
\text { collapse }\end{array}$ & $\begin{array}{l}\text { Proteinuria }>1 \mathrm{gm} / \mathrm{dl} \text {, S.Creatinine }>3.5 \\
\mathrm{mg} / \mathrm{dL}, \text { Elevated S Bilirubin }(>6 \mathrm{mg} / \mathrm{dl}) \text {, } \\
\text { ALT, AST }(>100 \mathrm{UL} / \mathrm{L}) \text {, } \\
\text { Thrombocytopenia }<20000 \text {, Haemolysis on } \\
\text { peripheral smear, clot observation time }>7 \\
\text { min. or any other test done which shows } \\
\text { deranged coagulation profile, Hypertensive } \\
\text { retinopathy }>\text { GRADE II, Abnormal ECG } \\
\text { (ST inversion, elevation/ arrhythmias, } \\
\text { Cerebral hemorrhage on CT scan }\end{array}$ \\
\hline \multicolumn{4}{|l|}{ Infections } \\
\hline $\begin{array}{l}\text { Severe systemic } \\
\text { infection or sepsis, } \\
\text { and others }\end{array}$ & $\begin{array}{l}\text { Cases of puerperal } \\
\text { sepsis, and post } \\
\text { abortion sepsis } \\
\text { Other severe } \\
\text { infections }\end{array}$ & $\begin{array}{l}\text { High grade fever (with/ } \\
\text { without chills and rigor) } \\
\text { Altered behavior } \\
\text { Breathlessness } \\
\text { Abdominal distension } \\
\text { Unconscious state }\end{array}$ & $\begin{array}{l}\text { Altered conscious state } \\
\text { Persistent rise in Temp }>39.2^{0} \mathrm{C} \text {, not } \\
\text { responding to routine treatment } \\
\text { Hypothermia Temp }<37^{0} \mathrm{C} \\
\text { Pulse Rate }>120 / \mathrm{min} \text {, Tachypnea }>20 / \mathrm{min} \\
\text { Coma, Bleeding from various sites }\end{array}$ \\
\hline \multicolumn{4}{|c|}{ Postpartum collapse } \\
\hline $\begin{array}{l}\text { Amniotic fluid } \\
\text { Embolism } \\
\text { Uterine Inversion }\end{array}$ & $\begin{array}{l}\text { Acute Collapse of } \\
\text { patient after } \\
\text { delivery }\end{array}$ & $\begin{array}{l}\text { Pulse not recordable } \\
\text { BP not recordable } \\
\text { Cardiorespiratory arrest }\end{array}$ & $\begin{array}{l}\text { Acute fall } \mathrm{Hb}<5 \mathrm{gm} \% \text { (fall in hemoglobin } \\
\text { so as to affect oxygen saturating) } \\
\text { Fall in oxygen saturation below } 90 \% \\
\mathrm{PaO}_{2} \mathrm{FiO}_{2}<<200 \\
\mathrm{PaCO}_{2}>50 \mathrm{~mm} \mathrm{Hg} \\
\text { Platelet }<20,000 \text { (Acute decline in platelet } \\
\text { count more significant) } \\
\text { Clot observation time }>7 \text { min. done which } \\
\text { proves deranged coagulation profile ECG- } \\
\text { Ischemic changes, ST inversion, Elevation }\end{array}$ \\
\hline \multicolumn{4}{|c|}{ Liver dysfunction / failure } \\
\hline $\begin{array}{l}\text { Acute fatty liver of } \\
\text { pregnancy } \\
\text { And others }\end{array}$ & $\begin{array}{l}\text { Convulsions } \\
\text { Altered behavior } \\
\text { Bleeding from } \\
\text { various sites } \\
\text { (nose, gums, IV } \\
\text { access ports, } \\
\text { varices) }\end{array}$ & $\begin{array}{l}\text { Unconsciousness } \\
\text { Deep jaundice } \\
\text { Hepatic flaps, tremors } \\
\text { Abnormal bleeding sites - } \\
\text { Haematuria, hematemesis, } \\
\text { haemoptesis, bleeding } \\
\text { gums etc. }\end{array}$ & $\begin{array}{l}\text { Elevated serum Bilirubin }(>6 \mathrm{mg} / \mathrm{DL}) \\
\text { Abnormal liver enzymes ALT, AST }>100 \\
\text { IU/L) } \\
\text { Abnormal ECG } \\
\text { Coagulation profile deranged } \\
\text { USG showing } \\
\text { Showing changes of Acute fatty liver } \\
\text { Fibro scan showing changes of acute fatty } \\
\text { liver }\end{array}$ \\
\hline \multicolumn{4}{|c|}{ Cardiac dysfunction/ failure } \\
\hline $\begin{array}{l}\text { RHD, CHD, } \\
\text { Cardiomyopathy } \\
\text { and others }\end{array}$ & $\begin{array}{l}\text { Breathlessness } \\
\text { specially at night } \\
\text { Palpitations } \\
\text { Chest pain }\end{array}$ & $\begin{array}{l}\text { tachycardia pulse }> \\
120 \mathrm{bpm} \\
\text { dyspnoea } \\
\text { Organic Murmurs }\end{array}$ & $\begin{array}{l}\text { Abnormal ECG } \\
\text { Abnormal echocardiography } \\
\text { X ray chest } \\
\text { (with shielding of abdomen) showing gross }\end{array}$ \\
\hline
\end{tabular}




\begin{tabular}{|c|c|c|c|}
\hline \multicolumn{4}{|c|}{$\begin{array}{l}\text { For diagnosis of near Miss, the patient should meet minimum } 3 \text { criteria: one each from 1) clinical findings } \\
\text { (either symptoms or signs, 2) investigations and } 3 \text { ) interventions done or any single criteria which signifies } \\
\text { cardiorespiratory collapse }\end{array}$} \\
\hline & orthopnea & $\begin{array}{l}\text { Cardimegaly } \\
\text { Signs of CCF/ LVF }\end{array}$ & $\begin{array}{l}\text { cardiomegaly) } \\
\text { Acid Base values } \\
\mathrm{PH}<7.35 \text { or }>7.45, \mathrm{PCO}_{2}>50 \text { or }<30 \\
\mathrm{mmHg}, \mathrm{PO}_{2} \text { arterial }<80 \mathrm{mmHg}\end{array}$ \\
\hline \multicolumn{4}{|l|}{ Severe anemia } \\
\hline & $\begin{array}{l}\text { Dyspnea } \\
\text { Palpitations } \\
\text { Syncopoal Attack } \\
\text { Altered conscious } \\
\text { state } \\
\text { Features of Sickle } \\
\text { cell crisis such as } \\
\text { bone pains, joint } \\
\text { pains, acute } \\
\text { abdominal pain etc. } \\
\text { Swelling over body }\end{array}$ & $\begin{array}{l}\text { Severe Pallor } \\
\text { Jaundice } \\
\text { Tachycardias pulse rate } \\
>120 / \text { min } \\
\text { Tachypnea > } 20 \text { / min } \\
\text { Tender, inflamed joints, } \\
\text { Sternal, tenderness, } \\
\text { Spleenomegaly } \\
\text { Anasarca, Ascites } \\
\text { Signs of congestive cardiac } \\
\text { failure } \\
\text { Bleeding tendencies }\end{array}$ & $\begin{array}{l}\text { Hemoglobin below } 5 \mathrm{mg} / \mathrm{dl} \\
\text { Hemoglobin status not able to maintain } \mathrm{O}_{2} \\
\text { Saturation of } 90 \% \\
\text { Platelet }<20000 \\
\text { Clot observation time }>7 \text { min. or any other } \\
\text { test done which proves deranged } \\
\text { coagulation profile } \\
\text { Elevated S Bilirubin }(>6 \mathrm{mg} / \mathrm{dl})\end{array}$ \\
\hline \multicolumn{4}{|c|}{ Respiratory dysfunction } \\
\hline $\begin{array}{l}\text { Asthma } \\
\text { Tuberculosis } \\
\text { Pneumonia }\end{array}$ & $\begin{array}{l}\text { Breathlessness / Air } \\
\text { hunger } \\
\text { High/ low grade } \\
\text { fever } \\
\text { Chronic weight loss }\end{array}$ & $\begin{array}{l}\text { Tachycardia }- \text { pulse rate }> \\
120 / \text { min } \\
\text { Tachypnea }>20 / \text { min } \\
\text { Orthopnea } \\
\text { abnormal chest signs } \\
\text { (ronchi, /Crepts, absent } \\
\text { breath sounds) } \\
\text { Signs or cardiorespiratory } \\
\text { failure, Cynosis, flaps }\end{array}$ & $\begin{array}{l}\text { Various lesions on chest } \mathrm{X} \text { ray (with } \\
\text { shielding of abdomen) specific to disease } \\
\text { Abnormal acid base values } \\
\mathrm{PH}<7.35 \text { or }>7.45 \\
\mathrm{PCO}_{2}>50 \text { or }<30 \mathrm{~mm} / \mathrm{hg} \\
\mathrm{PO}_{2} \text { arterial }<80 \mathrm{mmHg} \\
\mathrm{PO}_{2} \text { venous }<40 \mathrm{mmHg}\end{array}$ \\
\hline \multicolumn{4}{|c|}{ Cardiac Dysfunction } \\
\hline & $\begin{array}{l}\text { Breathlessness/ Air } \\
\text { hunger } \\
\text { Orthopnea } \\
\text { Palpitaitons } \\
\text { Paroxysmal } \\
\text { nocturnal dyspnea } \\
\text { Chest pain }\end{array}$ & $\begin{array}{l}\text { Tachycardia pulse rate } \\
>120 \text { min Bradycardia }> \\
40 \text { / min } \\
\text { Irregular pulse } \\
\text { Tachypnea }>40 \text { / min } \\
\text { Bradypnoea }<6 / \text { min } \\
\text { Organic murmurs } \\
\text { Cardiomegaly } \\
\text { Tender hepatomegaly } \\
\text { Signs of CCF / LVF, } \\
\text { pitting edema, raised JVP, } \\
\text { basal crepts etc. }\end{array}$ & $\begin{array}{l}\text { Abnormal ECG } \\
\text { Abnormal Echocardiography } \\
\text { Abnormal acid base values } \\
\mathrm{PH}<7.35 \text { or }>7.45 \mathrm{mmHg} \\
\mathrm{PCO}_{2}>50 \text { or }<30 \mathrm{mmHg} \\
\mathrm{PO}_{2} \text { artial }<80 \mathrm{mmHg}, \mathrm{PO}_{2} \text { venous }< \\
40 \mathrm{mmhg}\end{array}$ \\
\hline \multicolumn{4}{|c|}{ Hepatic Dysfunction } \\
\hline $\begin{array}{l}\text { Cirrhosis of liver } \\
\text { Portal } \\
\text { hypertension } \\
\text { Acute liver failure }\end{array}$ & $\begin{array}{l}\text { Yellowness of urine } \\
\text { / eyes other body } \\
\text { parts } \\
\text { Convulsions } \\
\text { Altered behavior } \\
\text { bleeding from } \\
\text { various sites } \\
\text { (nose, gums, IV } \\
\text { access ports, } \\
\text { varices) }\end{array}$ & $\begin{array}{l}\text { Deep jaundice } \\
\text { Hepatic flaps/ tremors } \\
\text { Abnormal bleeding sites - } \\
\text { heamaturia, haematemesis, } \\
\text { haemoptysis, bleeding } \\
\text { gums etc. } \\
\text { Abnormal bleeding from } \\
\text { nose, gums, I/V sites, } \\
\text { varices } \\
\text { Hepatomegaly Ascites }\end{array}$ & $\begin{array}{l}\text { Elevated Serum Bilirubin }(>6 \mathrm{mg} / \mathrm{dl}) \\
\text { Abnormal lover enzymes ALT, AST }(>100 \\
\text { IU/L) } \\
\text { Abnormal ECG } \\
\text { Clot observation time }>7 \mathrm{~min} / \text { or any other } \\
\text { test done which proves deranged } \\
\text { coagulation profile } \\
\text { Imaging modalities showing hepatomegaly, } \\
\text { splenomegaly and other abnormalities }\end{array}$ \\
\hline \multicolumn{4}{|c|}{ Endocrinal disorders Ketacidosis } \\
\hline & $\begin{array}{l}\text { Altered conscious } \\
\text { state } \\
\text { Breathlessness / air } \\
\text { hunger }\end{array}$ & $\begin{array}{l}\text { Features of circulatory } \\
\text { collapse } \\
\text { Neurological deficit like } \\
\text { muscular weakness, }\end{array}$ & $\begin{array}{l}\text { Ketoacidosis } \mathrm{pH}<7.35 \mathrm{RBS}>200 \mathrm{~g} / \mathrm{dl} \\
\text { Abnormal ECG } \\
\text { Electrolyte, Imbalance }(\mathrm{Sr} \mathrm{Na}<129 \mathrm{~K}<3.2 \\
->5.5\end{array}$ \\
\hline
\end{tabular}




\begin{abstract}
For diagnosis of near Miss, the patient should meet minimum 3 criteria: one each from 1) clinical findings (either symptoms or signs, 2) investigations and 3 ) interventions done or any single criteria which signifies cardiorespiratory collapse

\begin{tabular}{|c|c|c|c|}
\hline \multirow{4}{*}{$\begin{array}{l}\text { Thyroid Crisis } \\
\text { Thyrotoxicosis } \\
\text { Thyroid storm }\end{array}$} & \multirow{2}{*}{$\begin{array}{l}\text { Palpitations, } \\
\text { Convulsions } \\
\text { Palpitations }\end{array}$} & \multicolumn{2}{|l|}{$\begin{array}{l}\text { paresis, plegia } \\
\text { Altered consciousness, } \\
\text { coma }\end{array}$} \\
\hline & & Altered Consciousness & Sr. $\mathrm{T}_{4}$ Elevated (>200 IU) \\
\hline & Convulsions & Coma & Low TSH $(<0.2$ IU $)$ \\
\hline & $\begin{array}{l}\text { Bladder / Bowel } \\
\text { dysfunction }\end{array}$ & $\begin{array}{l}\text { Tachycardia pulse }>120 \\
\text { bpm }\end{array}$ & $\begin{array}{l}\text { Ischaemic changes on ECG } \\
\text { Elevated Vinyl mandilic acid }\end{array}$ \\
\hline
\end{tabular}

Note: Any Intervention: ICU admission requiring resuscitative (CAB) or cardio respiratory support and or Endotracheal intubation ,Use of blood \& blood pro ducts transfusion $>90 \mathrm{mllkg}$ body weight / > 5 units of blood), Use of cardiotonics/Vaso pressors ( Mephentine / Dobutamine/ dopamine etc), Cirulatory collapse requiring emergency surgery, Dialysis- use of Adrenaline Renal Dialysis pertonela/ hemo Dialysis, Use of fourth generation Anti Biotic and others.
\end{abstract}

\section{RESULTS}

This is a retrospective study done in department of Obstetrics and Gynecology in GMC associated with DHS Shivpuri MP. The study was done during a period from 1st January 2018 to 30 April 2019. During this period 13849 ANC cases were admitted in hospital, of which 11348 patients were delivered, 2342 patients required lower segment caesarean section (LSCS), giving a LSCS rate of $20.63 \% .11087$ live birth were there. 159 cases were diagnosed as near miss.

I calculated intra Hospital maternal near miss incidence ratio (number of maternal near miss cases per 1000 live birth) (MNM IR = MNM/ LB). In my study the hospital maternal near miss incidence ratio was $14.34 \%$ per 1000 live birth. Table 2 shows the demographic details of these 159 women. In this study most women $(55.34 \%)$ were of age of less than 25 years, $(40.88 \%)$ were primipara, $(55.97 \%)$ were unbooked and were admitted in emergency.

Literacy level was low and majority $(64.15 \%)$ were from low socio-economic group. Majority of the patients $(50.94 \%)$ were in third trimester. $(44.02 \%)$ cases were referred in cases and most of them $(61.00 \%)$ were rural.
Table 3 shows summarizes the causes of near miss in my study group. In this study we found the most common morbidity was (30.18\%) hypertensive disorder of pregnancy.

Figure 1: In this study very severe anemia (13.20\%) was the commonest medical disorder found in near miss cases these women presented commonly with generalized edema weakness dyspnoea and unable to perform routine work.

Author could not find first delay (delay before seeking health care) and second delay (delay in reaching to health facility) because it was a retrospective study, but we tried to find third delay (delay in receiving care).

The median time taken to get clinical intervention among cases was 20-40 minutes after admission. Reduced time of intervention was due to preparedness and promptness of health staff after various training under LaQshya Programme. There was delay in receiving adequate and appropriate treatment (delay 3) in only in 17 (10.69\%) women. Figure 2: During this period there was 8 maternal deaths were happened there. The most common cause of death was post-partum haemorrhage (37.5\%).

Table 2: Socio- demographic variables, parity and gestational age of participants.

\begin{tabular}{|c|c|c|}
\hline Variables & Number & Percentage \\
\hline \multicolumn{3}{|c|}{ Age of mother (years) } \\
\hline $15-20$ & 07 & 4.40 \\
\hline $21-25$ & 88 & 55.34 \\
\hline $26-35$ & 47 & 29.55 \\
\hline$>35$ & 17 & 10.69 \\
\hline \multicolumn{3}{|l|}{ Parity } \\
\hline 0 & 65 & 40.88 \\
\hline 1 & 60 & 37.73 \\
\hline 2 & 21 & 13.20 \\
\hline$>2$ & 13 & 8.17 \\
\hline \multicolumn{3}{|c|}{ Gestational age } \\
\hline$<12$ weeks & 15 & 9.43 \\
\hline
\end{tabular}




\begin{tabular}{|lll|}
\hline Variables & Number & Percentage \\
\hline $13-28$ wks & 07 & 4.40 \\
\hline $29-36$ wks & 40 & 25.15 \\
\hline $37-40$ wks & 81 & 50.94 \\
\hline$>40$ wks & 09 & 5.66 \\
\hline Postpartum & 07 & 4.40 \\
\hline Booking status & 70 & \\
\hline Booked & 89 & 44.025 \\
\hline Unbooked & & 55.97 \\
\hline Literacy & 80 & 50.31 \\
\hline Illiterate & 79 & 49.68 \\
\hline Literate & & \\
\hline Socioeconomic status & 102 & 64.15 \\
\hline Low & 52 & 32.70 \\
\hline Middle & 5 & 3.144 \\
\hline High & & \\
\hline Residence & 62 & 38.99 \\
\hline Urban & 97 & 61.00 \\
\hline Rural & & \\
\hline Referral status & 89 & 55.97 \\
\hline Self & 70 & 44.02 \\
\hline Referred from a health facility & & \\
\hline
\end{tabular}

Table 3: Causes of near miss cases $n=159$.

\begin{tabular}{|c|c|c|c|}
\hline Diagnosis & & $\mathbf{N}$ & $\%$ \\
\hline $\begin{array}{l}\text { Hypertensive disorders in } \\
\text { pregnancy }\end{array}$ & $\begin{array}{l}\text { Chronic hypertension. severe PIH, severe preeclampsia with signs of } \\
\text { organ dysfunction / eclampsia with organ dysfunction, HELP syndrome }\end{array}$ & 48 & 30.18 \\
\hline \multirow{9}{*}{ Severe hemorrhage } & Hemorrhage due to RPOC and inevitable abortion & \multirow{9}{*}{44} & \multirow{9}{*}{27.67} \\
\hline & Ruptured ectopic pregnancy with severe anemia and shock & & \\
\hline & Placenta praevia & & \\
\hline & Placental abruption & & \\
\hline & intra operative hemorrhage & & \\
\hline & Rupture uterus & & \\
\hline & Severe post-partum hemorrhage & & \\
\hline & Hemorrhage due to retained placenta & & \\
\hline & Genital tract injuries and large hematomas & & \\
\hline \multirow{4}{*}{$\begin{array}{l}\text { Severe systemic infection } \\
\text { or sepsis }\end{array}$} & Septic abortion & \multirow{4}{*}{11} & \multirow{4}{*}{6.91} \\
\hline & Chorio amnionitis & & \\
\hline & Puerperal sepsis & & \\
\hline & $\begin{array}{l}\text { Postsurgical procedure severe infection (E.g. Cesarean section, } \\
\text { laprotomy, evacuation, manual removal of placenta and others. }\end{array}$ & & \\
\hline Labour related disorders & $\begin{array}{l}\text { Prolonged and obstructed labour with complications, Rupture uterus and } \\
\text { others. }\end{array}$ & 7 & 4.40 \\
\hline Postpartum collapse & Amniotic fluid embolism, inversion of uterus & 3 & 1.88 \\
\hline Medical disorders & & & 24.47 \\
\hline Very aevere anemia & ( $>3$ blood transfusion / PRBCs and multiple doses of iron sucrose) & 21 & 13.20 \\
\hline $\begin{array}{l}\text { Cardiovascular } \\
\text { dysfunction }\end{array}$ & Valvular disease, arrhythmia, cardio myopathy, infarction, & 3 & 1.88 \\
\hline Respiratory dysfunction & $\begin{array}{l}\text { ARDS } \\
\text { Pulmonary edema } \\
\text { Post-operative pneumonia and others } \\
\text { Severe tuberculosis with respiratory failure. }\end{array}$ & 7 & 4.40 \\
\hline Renal dysfunction & Oliguria needed multiple doses of diuretics/ dialysis acute renal failure & 4 & 2.51 \\
\hline Coagulation dysfunction & DIC & 1 & 0.62 \\
\hline
\end{tabular}




\begin{tabular}{|llll|}
\hline Diagnosis & & N & $\%$ \\
\hline Hepatic dysfunction & Acute fatty liver of pregnancy and others & 1 & 0.62 \\
\hline Neurological dysfunction & Intracranial hemorrhage, non-eclamptic seizures, & 1 & 0.62 \\
\hline Endocrine disorders & Diabetic keto acidosis, Thyroid crisis & 1 & 0.62 \\
\hline Anesthetic complications & Allergic reaction, total spinal and failed intubation & 5 & 3.144 \\
\hline Incidental/ Accidental Disorders E.g. Surgical including latrogenic, Trauma, Violence, etc. & 2 & 1.25 \\
\hline Total & & 159 & $100 \%$ \\
\hline
\end{tabular}

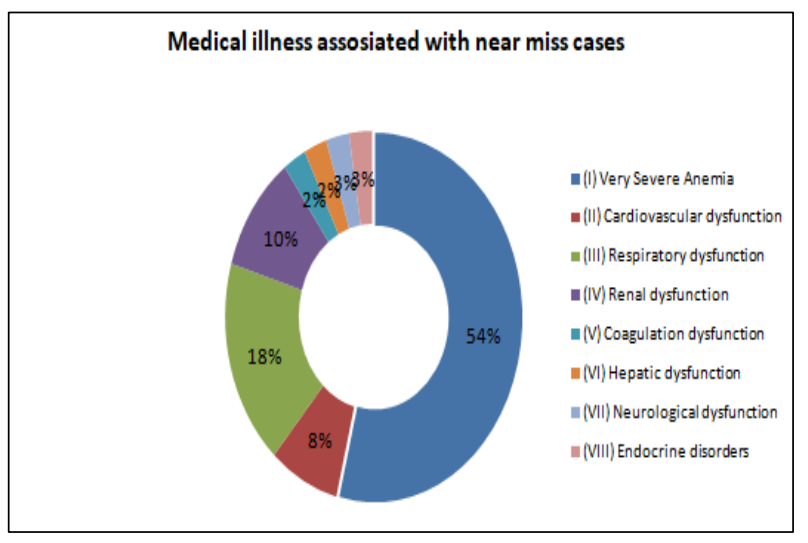

Figure 1: Medical disorders, as a cause of near miss cases.

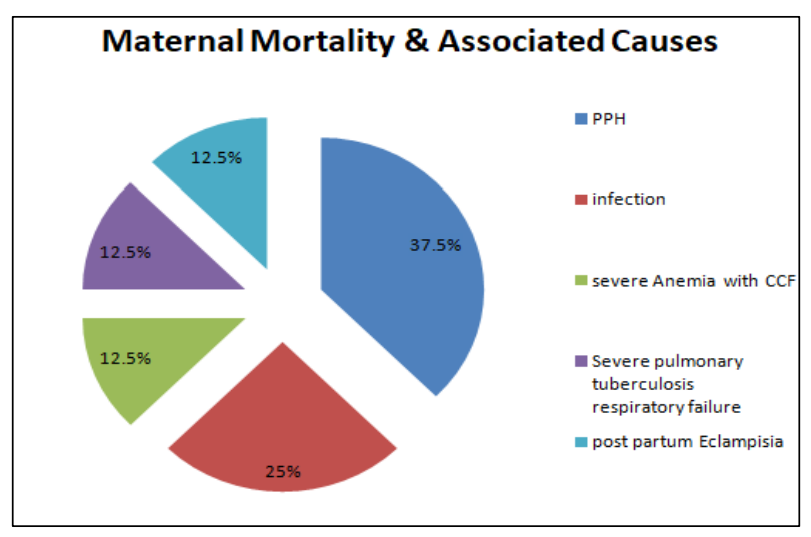

Figure 2: Two Causes of maternal mortality.

\section{DISCUSSION}

In this study- $55.34 \%$ of women were in age group of 21-25, 55.97\% were unbooked, $40.88 \%$ were primipara, $50.94 \%$ were term and $64.15 \%$ were of low income group. My study was comparable with one another study, in which $53.3 \%$ of women with near miss in their hospital were in the age group of 21-25 year, $66.6 \%$ was primipara, $73.3 \%$ were term and $60 \%$ were from low income group. ${ }^{6}$ In one another study most women $67.85 \%$ were age of $<25$ year, $64.28 \%$ were unbooked and were admitted in emergency, literacy rate was low and majority was from low social economic group. ${ }^{7}$
The MNM IR was $14.34 \%$ in this study which is comparable to study of Roopa PS et al, with maternal near miss incidence ratio was $17.8 / 1000$ live birth. ${ }^{8}$ The incidence of SAMM was 15 and 17.8 per 1000 live birth in study of Moraes et al, and Ps et al, respectively. ${ }^{9,10}$ The incidence of severe maternal morbidity was 3.3/1000 deliveries in study by Chhabra $\mathrm{P}$ et al, and Waterstone et al, reported a severe obstetric morbidity rate of 12.0/1000 live births, which was lower than my study. ${ }^{11,12}$ In this study he found the most common morbidity was (30.18\%) hypertensive disorder of pregnancy, second one was $(27.67 \%)$ of severe hemorrhage. Upadhyaya and Chaudhary, Moracs et al, and Huseyin et al, also reported the hypertensive disorder in pregnancy as leading cause of maternal illness. ${ }^{13-15}$ Also, the Study by Souza JP et al, had incidence of severe preeclampsia in $36.3 \%$, Eclampsia 9.7\%, HELLP syndrome 5.6\%. severe hemorrhage $10.5 \%$, severe sepsis $6.4 \% .^{16}$

Author study was not comparable with study of While Taly et al, Roost et al, and Manandhar et al, they reported hemorrhage $60 \%, 48 \%$ and $41.66 \%$ as most common cause of SAMM (near miss) respectively. ${ }^{17,18}$

In this study severe anemia $(13.20 \%)$ was the most common medical disorder found in near miss cases. The other studies form our country have also reported anemia as an important cause and contributor to maternal mortality and severe maternal morbidity. ${ }^{19}$ Even after implementation of different programs for controlling iron deficiency anemia, the magnitude of this problem is still high.

During the study period 8 patients were died, out of which $3(37.5 \%)$ patients were died due to PPH. It is comparable with study conducted by Mehta $\mathrm{M}$ et al, showed major cause of death in his study was hemorrhage. ${ }^{20}$

There was delay in receiving adequate and appropriate treatment (delay 3) only in $17(10.69 \%)$ near miss cases, that was lower than one another study in which delay was in $(21.8 \%)$ women. ${ }^{21}$

Globally, there has been a paradigm shift in the maternal care strategy since the 1990's. In India also there has been a policy change with promotion of institutional 
births, births by skilled birth attendants and provision of Emergency obstetric Care. ${ }^{22}$

Reduction of maternal and neonatal morbidity and mortality is one of the key objectives of the National Health Mission. (NHM), so that India achieve the SDG target of MMR of less than 70 per Lakh live Births.

The Janani Suraksha Yojana (JSY) a cash incentive scheme has been initiated to promote institutional deliveries. A recent study on impact of JSY has shown an increase in institutional deliveries among the vulnerable and high risk cases such as pre-eclampsia, eclampsia, hemorrhage, severe anemia etc. Several other interventions and programmed aids such as $\mathrm{MNH}$ Toolkit, Standardizations guidelines for the labour Rooms, 'Dakshata', MDR and CDR guidelines, National Quality Assurance Standards, establishment of skill labs, PMSMA etc. to achieve substantial reduction in maternal morbidity, maternal motility and newborn morbidity.

Ministry of Health and Family Welfare has recently launched the program 'LaQshaya' aimed at further improving quality of care pregnant women in labour room, maternity operation Theatre and obstetrics intensive Care Units (ICUs) and High Dependency Units (HDUs). ${ }^{23}$ The LaQshaya program is being implemented at all Medical College Hospitals, District Hospitals and First Referral Unit (FRU), and Community Health Center (CHCs) and will benefit every pregnant woman and new born delivering in public health institutions.

The program aimed at implementing 'fast track' interventions for achieving tangible results within 18 months. Under the initiative, a multi - pronged strategy has been adopted such as improving infrastructure up gradation, ensuring availability of essential equipment, providing adequate human resources, capacity building of health care workers and improving quality processes in the labour room. To strengthen critical care in Obstetrics, dedicated obstetric ICUs at Medical Colleges Hospitals level and Obstetric HDUs at District Hospitals are operationalized under LaQshya program. The quality of improvement in labour room and maternity OT will be assessed through NQAS (National Quality Assurance Standards).

\section{The Objectives of LaQshya initiative}

- To reduce maternal and newborn mortality and morbidity due to $\mathrm{APH}, \mathrm{PPH}$, retained placenta, preterm, preeclampsia and eclampsia, obstructed labour, puerperal sepsis, newborn asphyxia, and sepsis, etc.

- $\quad$ To improve Quality of care during the delivery and immediate post-partum care, stabilization of complications and ensure timely referrals, and enable and effective two-way follow-up system.

- To enhance satisfactions of beneficiaries visiting the health facilities and provide Respectful Maternity
Care (RMC) to all pregnant women attending the public health facility.

Efforts geared towards improvements in the management of near-miss morbidities would definitely go a long way in reducing the present maternal mortality ratio.

\section{CONCLUSION}

According to author study hypertension and hemorrhagic disorders are the leading cause of maternal near miss. Efforts must be made to improve maternal care for hypertension and hemorrhagic disorders. Other lifethreatening conditions like severe anemia, infection, and uterine rupture should also be taken care of. It is well known that complication during pregnancy and child birth can occur at any point of time, and it is important to ensure that readiness in terms of infrastructure, HR, equipment etc, for timely management of complications are available at all the basic and emergency obstetric care health facilities. A regular audit of near miss cases and reporting of these cases to higher authorities should be done so that appropriate action can be taken at all care levels. Proper data management must be done for quick and effective treatment. Prompt diagnosis, appropriate action and timely intervention will reduce maternal morbidity and mortality.

\section{ACKNOWLEDGMENTS}

Authors would like to thank Dr. Dilip Jain, consultant pathologist for helping me in this study.

Funding: No funding sources

Conflict of interest: None declared

Ethical approval: Not required

\section{REFERENCES}

1. Kaur R, Roy D, Aggarwal P, Nautiyal R, Chaturvedi J, kakkar R. Application of WHO Near miss tool Indicates Good Quality of Maternal Care in Rural Healthcare Setting in Uttarakhand, Northern India: J Clin Diagn Res. 2016;10(1):LC10-3.

2. Pattinson RC, Say L, Souza JP, Broek NV, Rooney C. WHO Working Group on maternal Mortality and Morbidity Classifications. WHO maternal death and near miss classifications. Bull World Health Organ. 2009;87(10):734.

3. Tallapureddy S, Velagaleti R, Palutla H, Satti CV; “ Near Miss" Obstetric events and maternal mortality in a Tertiary Care Hospital; Indian J Public Health 2017 Oct- Dec; 61(4):350.

4. Aboubakr Elnashar; Behna University Hospital, Egypt, Maternal near miss - slideshare, 30 Oct 2018. Available at: http://www.slideshare.net.elnashar. 
5. Mishra C.K. Maternal Near miss review operational guidelines. December 2014. Annexure 2: 35-40.

6. Sharma S, Aherwar R, Jawadhe S. Retrospective study of maternal near misses in a tertiary care instate: international Journal of Reproduction, contraception, Obstetrics and Gynecology. 2016;5(4)1114-8.

7. Kaur N, Aryal S. Pattern of Severe Acute Maternal Morbidity in a Tertiary Care Institute: J. Lumbini Med. Coll. 2015;3(2):45-9.

8. Roopa PS, Verma S, Rai L, Kumar P, Murlidhr V, Pai, et. Al "Near Miss" Obstetric Events and Maternal Deaths in a Tertiary Care Hospital: An Audit Journal of Pregnancy (2013).

9. Moraes AP, Barreto SM, Passos VM, Golino PS, Costa JA, Vasconcelos MX. Incidence and main causes of severe maternal morbidity in Sáo Lusis, Maranháo, Brazil: A longitudinal study Sao Paulo Med J. 2011;129:146-52.

10. Sangeeta G, Leena W, Taru G, Sushma K , Nupur $\mathrm{G}$, Amrita $\mathrm{P}$, et al. Evaluation of severe maternal outcomes to assess quality of maternal health care at a tertiary center. J Obstet Gynaecol India 2015;65:23-7.

11. Chhabra P, Guleria K, Saini N, Anjur KT. Pattern of severe maternal morbidity in a tertiary hospital of Delhi, India: a pilot study. 2008;38(4):201-4.

12. Waterstone $M$, Bewley $S$, Wolfe $C$. Incidence and predictors of severe obstetric morbidity: case- control study. MBJ. 2001;322(7294):1089-4.

13. Upadhyaya I, Chaudhary P. Severe acute maternal morbidity and intensive care in Paropkar maternity and women's hospital. NJOG. 2013;8(2):38-41.

14. Moraes AP, Barreto SM, Passos VM, Golino PS, Costa JA, Vasconcelos MX. Incidence and main causes of severe maternal morbidity in Sao Luis Maranhao, Brazil: A longitudinal Study. Sao Paulo Med J. 2011;129(3):146-52.

15. Huseyin C, Cihan K, Ramzan A, Ziya YY, Murat E, Levent Y. Near miss obstetric cases: 4 years experience of a tertiary center. Gynecol Obstet Reprod Med. 2013;19(1):19-22.

16. Sauza JP, Cecatti JG, Parpinelli MA, Serruya SJ, AmaraI E. Appropriate criteria for identification of near miss maternal morbidity in tertiary care facilities: A cross sectional study BMC pregnancy and childbirth. 2007;7(1):20.

17. Taly A, Gupat S, Jain N. Maternal Intensive care and near miss mortality in obstetrics. J Obstet Gynecol India. 2004;54(5):478-82.

18. Roost M, Altamirano VC, Liljestrand J, Essen B. Priorities in emergency obstetric care in Bolivia Maternal mortality and near miss morbidity in metropolitan La Paz. BJOG. 2009;116(9):1210-7.

19. Daru J, Zamora J, Fernández-Félix BM, Vogel J, Oladapo OT, Morisaki N, et al. Risk of maternal mortality in women with severe anaemia during pregnancy and post-partum: a multilevel analysis. The Lancet Global Health. 2018;6(5):e548-54.

20. Mehta M, BAvarva N. Facility base maternal Death Review at Tertiary care Hospital: A small Effort ot Explore Hidden facts. Appl Med Res. 2016;1(4):126-9.

21. Reena RP, Radha KR. Factors associated with maternal near miss: A study from Kerala. Indian J Public Health. 2018;62(1):58-60.

22. National program Implementation plan $\mathrm{RCH}$ Phase II- program document. Available at: http://mohfw.nic.in/NRHM/RCH/guidelines/PIP/Rev III.pdf. Accessed on 21 Feb 2012.

23. LaQshya: Labour room Quality Improvement Initiative In: National Health Mission; Ministry of Health \& Family Welfare Government of India. 2017:03,12-14.

Cite this article as: Jain U. A study on maternal near miss cases in government medical college Shivpuri, India. Int J Reprod Contracept Obstet Gynecol 2019;8:3047-55. 